\title{
State-to-state methane-surface scattering as a probe of catalytic activity
}

\author{
Jörn Werdecker, ${ }^{1}$ Bo-Jung Chen, ${ }^{1}$ Maarten E. Van Reijzen, ${ }^{1}$ Azar Farjamnia, ${ }^{2}$ Bret Jackson $\odot,{ }^{2}$ and Rainer D. Beck $\odot^{1,},{ }^{*}$ \\ ${ }^{1}$ Laboratoire de Chimie Physique Moléculaire, École Polytechnique Fédérale de Lausanne, CH-1015 Lausanne, Switzerland \\ ${ }^{2}$ Department of Chemistry, University of Massachusetts, Amherst, Massachusetts 01003, USA
}

(Received 12 June 2020; accepted 28 October 2020; published 18 November 2020)

\begin{abstract}
Quantum state-resolved scattering experiments for methane molecules colliding with a catalytically active nickel surface are compared to scattering from a nickel surface passivated by a single layer of graphene. The vibrational state distribution of the scattered methane is observed to differ dramatically for the two surfaces. Quantum-mechanical inelastic scattering calculations show that these differences are related to the catalytic activity of the surface impact site. Our results demonstrate how inelastic scattering can be used to probe the reactive potential-energy surfaces of molecule-metal systems important to heterogeneous catalysis.
\end{abstract}

DOI: 10.1103/PhysRevResearch.2.043251

\section{INTRODUCTION}

Uncovering the dynamics of a chemical reaction between a gas-phase molecule and a solid surface is key to understanding and predicting heterogeneous catalysis, a process of great economic and ecological value. For example, solid-phase catalysts are involved in the production of the fuel that powers the combustion engine in our automobiles as well as in cleanup of its exhaust gases. The search for more efficient catalysts is therefore of great economic and ecological importance and can undoubtedly benefit from a detailed, microscopic understanding of the dynamical processes occurring on catalytically active surfaces.

Most studies of the reaction dynamics at the gas-surface interface have focused on reactive events by detecting reaction products and measuring how reaction rates depend on the energy and internal state of incident molecule [1,2]. Consider the dissociation of methane on transition-metal catalysts; of particular interest since it is the rate-limiting step in steam reforming, a process of great importance for the chemical industry. During the last two decades, this reaction has been extensively explored, both experimentally and computationally, revealing nonstatistical dynamics that include mode specificity [3], bond selectivity [4,5], and steric effects [6]. However, much less is known about the fate of those molecules that fail to react during their collision with the catalyst surface and instead scatter back inelastically into the gas phase. This is especially true for polyatomic molecules such as methane because of a lack of generally applicable and highly sensitive detection techniques that can provide information on the vibrational and rotation quantum states of

\footnotetext{
*rainer.beck@epfl.ch

Published by the American Physical Society under the terms of the Creative Commons Attribution 4.0 International license. Further distribution of this work must maintain attribution to the author(s) and the published article's title, journal citation, and DOI.
}

the scattered species. The lack of scattering data is unfortunate because it leaves us blind to important features of the interactions governing reactions. As we demonstrate in this paper, those state distributions carry signatures of the rich dynamics that unfold as the incident molecule makes its way along the reaction path, before being repelled back into the gas phase. By contrast, molecules that succeed in reacting on the surface lose all memory of those dynamics as they interact with the plethora of surface states.

Recently, we have implemented a state-resolved method for detecting molecules scattered from surfaces based on a cryogenic bolometer and quantum state-specific infrared laser tagging $[7,8]$. The method, which we call bolometer infrared laser tagging (BILT), enables the state-resolved detection of methane or any molecule with an infrared-active vibrational mode and a rotationally resolved infrared spectrum. The combination of quantum state-specific preparation of the reactant molecules in the incident molecular beam by infrared laser pumping, with state-resolved BILT detection of the scattered species, enables so-called "state-to-state" scattering experiments. They provide highly detailed information about the scattering dynamics and energy transfer between the molecular and surface degrees of freedom. When state-to-state scattering experiments are accompanied by a quantum-dynamics simulation on a realistic potential energy hypersurface calculated through density-functional theory, it becomes possible to develop a complete and detailed picture of the different inelastic and reactive processes that control the outcome of a molecule/surface collision.

Here, we report dramatic changes in the product state distribution observed for state-to-state scattering of methane from clean and graphene-covered $\mathrm{Ni}(111)$ surfaces. The experimental findings are compared to fully quantummechanical scattering calculations, which relate the amount of intramolecular vibrational energy redistribution (IVR) to the catalytic activity of the impact site; i.e., the ability of a particular surface site to activate, or break, a $\mathrm{C}-\mathrm{H}$ bond. We thus demonstrate how inelastic scattering can be used to probe the reactive potential-energy 
surfaces of molecule-metal systems important to heterogeneous catalysis.

\section{METHODS}

The state-to-state scattering experiments were carried out in a previously described $[9,10]$ molecular beam-surface science apparatus. Briefly, a continuous molecular beam of methane was produced by supersonic expansion of $\mathrm{CH}_{4}$ seeded in He. Quantum state-specific preparation of the surface incident methane was achieved by infrared laser excitation of the $\mathrm{CH}_{4}$ molecules in the molecular beam using a tunable continuous-wave optical parametric oscillator system (IR-OPO). The state-prepared methane collided with a clean or graphene-covered $\mathrm{Ni}(111)$ sample in an ultrahighvacuum chamber. Scattered methane molecules were detected with quantum state resolution by a highly sensitive liquid He-cooled bolometer in combination with state-specific laser tagging using a second IR-OPO system by virtue of the BILT technique, as we have demonstrated in a recent publication [8]. Details of the experimental methods are described in the Supplemental Material [11].

To examine the vibrationally inelastic scattering of $\mathrm{CH}_{4}$ we use a quantum approach that has successfully described the dissociative chemisorption of methane on $\mathrm{Ni}$ and Pt surfaces [12-15]. This method, which provides a very accurate treatment of the molecular vibrations, is described in these earlier works and the Supplemental Material [11], and we summarize only the essential ideas here.

We consider normal incidence and treat the center-of-mass motion of $\mathrm{CH}_{4}$ parallel to the surface (coordinates $X$ and $Y$ ) using a sudden approximation. That is, we compute the scattering probabilities for impact on a particular surface site, keeping $X$ and $Y$ fixed, then repeat this for several other impact sites, averaging the results. This has worked well for similar systems $[16,17]$. For each impact site we locate the minimum energy path (MEP) as the molecule moves towards the surface. To compute the energy we use VASP, the Vienna $A b$ initio Simulation Package, developed at the Institut für Materialphysik of the Universität Wien [18-22]. Exchangecorrelation effects are included using a Specific Reaction Parameter functional shown to accurately describe the dissociative chemisorption of methane on $\mathrm{Ni}$ and $\mathrm{Pt}$ surfaces [23].

At several dozen points along each MEP we compute the total energy $V_{0}(s)$, where $s$ is the distance along the path in mass-weighted coordinates. We also compute and diagonalize the Hessian to find the normal vibrational coordinates $Q_{k}$ and corresponding frequencies, $\omega_{k}(s)$, describing motion orthogonal to the MEP in the harmonic approximation. Ignoring anharmonic terms we write our potential-energy surface, for a given MEP, in the reaction path coordinates $s$ and $Q_{k}$, as [24]

$$
V=V_{0}(s)+\sum_{k=1}^{9} \frac{1}{2} \omega_{k}^{2}(s) Q_{k}^{2} .
$$

This harmonic treatment is accurate for the strongly bound stretching and bending vibrations of the methane, modes 1 through 9 , but it is not a good description of $X, Y$, or rotational motion. As noted, $X$ and $Y$ are treated with a sudden model. The rotational degrees of freedom are allowed to evolve adi- abatically, reasonable at these low energies. The remaining 10 degrees of freedom, $s$ and the $Q_{k}$, are described by expanding our molecular wave function in a vibrational basis $[11-13,15,24]$ :

$$
\Psi(t)=\sum_{\mathbf{n}} \chi_{\mathbf{n}}(s ; t) \Phi_{\mathbf{n}}\left(\left\{Q_{k}\right\} ; s\right) .
$$

The $\Phi_{\mathbf{n}}$ are vibrationally adiabatic harmonic oscillator eigenfunctions that depend parametrically on $s$, and $\mathbf{n}$ labels each vibrational state. As we time evolve the wave packets, $\chi_{\mathbf{n}}$, nonadiabatic couplings create transitions between different vibrationally adiabatic states. The backscattered wave packets are analyzed to extract energy-resolved vibrational state probabilities [12].

\section{RESULTS}

Figure 1 shows a comparison of two BILT spectra recorded when state-prepared $\mathrm{CH}_{4}\left(\nu_{3}=1, J=1\right)$ is scattered from a bare $\mathrm{Ni}(111)$ and from a graphene-covered $\mathrm{Ni}(111)$ surface, respectively. The quantum-state population of the scattered $\mathrm{CH}_{4}$ is encoded in these spectra with each line corresponding to a vibrational transition originating from a rovibrationally excited quantum state that was populated by the moleculesurface collision. In order to record BILT spectra such as the ones shown in Fig. 1, the infrared tagging laser was scanned over a spectral region covering both $\nu_{3} \rightarrow 2 \nu_{3}$ and $v_{1} \rightarrow v_{1}+v_{3}$ hot-band transitions [11]. Vibrationally excited states at lower energy than $v_{1}$ were also probed and found to contain no detectable population irrespective of which of the two surfaces was present.

There is striking difference between the BILT spectra obtained for collisions with bare $\mathrm{Ni}(111)$ and those with $\mathrm{Gr} / \mathrm{Ni}(111)$. When scattering from the bare $\mathrm{Ni}(111)$ surface [8], we detect both vibrationally elastic scattering into the $v_{3}$ vibrational state as well as vibrationally inelastic scattering into the $v_{1}$ vibrational state, which is approximately $100 \mathrm{~cm}^{-1}$ lower in vibrational energy than $v_{3}$. On the other hand, when scattering from $\mathrm{Gr} / \mathrm{Ni}(111)$, all lines coming from the $v_{1}$ vibrational state are absent and we detect only vibrationally elastic scattering into different rotational levels of the $v_{3}$ vibrational state. This indicates that a single layer of graphene deposited on the $\mathrm{Ni}(111)$ surface completely eliminates the $v_{3} \rightarrow v_{1}$ vibrational energy redistribution that was easily detected with a branching ratio of $40 \%$ for scattering from the bare Ni(111) surface [8].

Our quantum dynamics simulations described below confirm that the absence of surface-induced IVR is due to changes in the $\mathrm{CH}_{4}$-metal interaction when a single layer of graphene is deposited on top of the $\mathrm{Ni}(111)$ surface. Furthermore, the simulations suggest that the propensity for $v_{3} \rightarrow v_{1}$ IVR scales with the chemical reactivity of the surface site where the scattering takes place.

Besides information on surface-induced IVR, the recorded spectra also contain information on the molecule-surface rotation energy transfer during the direct scattering event. The incident $\mathrm{CH}_{4}\left(\nu_{3}=1, J=1\right)$ is rotationless in the laboratory frame since its total angular momentum $(J=1)$ is solely due to vibrational angular momentum $(l=1)$ intrinsic in the triply degenerate $v_{3}$ vibration. Following scattering from the 

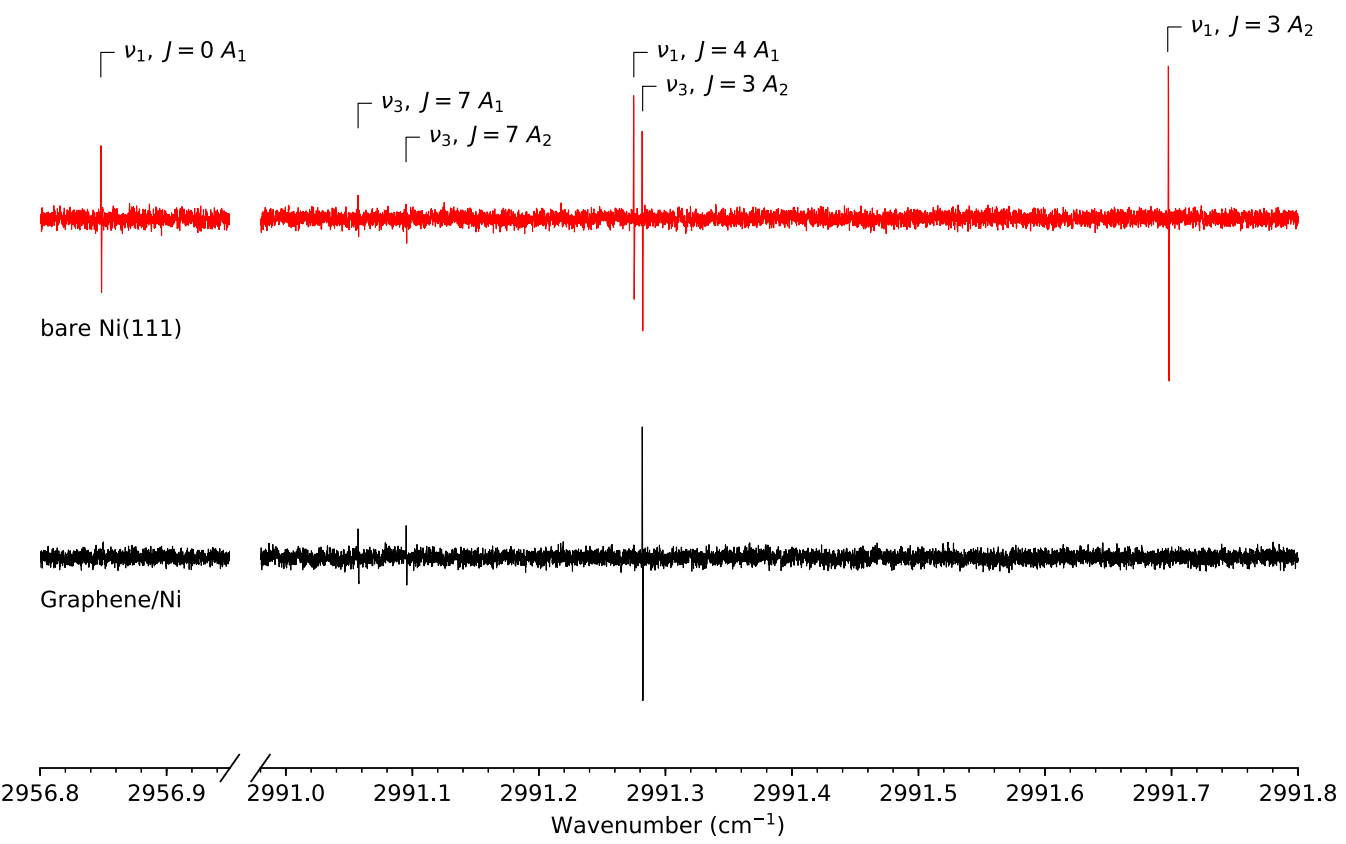

FIG. 1. Examples of BILT spectra obtained for scattering of state-prepared $\mathrm{CH}_{4}\left(v_{3} ; J=1\right)$ from clean $\mathrm{Ni}(111)$ (top trace in red) and graphene-covered $\mathrm{Ni}(111)$ (lower trace in black). Each spectral line marks a specific rovibrational quantum state of the scattered $\mathrm{CH}_{4}$ as indicated by the labels. For scattering from $\mathrm{Ni}(111), 40 \%$ of the incident $\mathrm{CH}_{4}\left(v_{3} ; J=1\right)$ molecules undergo $v_{3} \rightarrow v_{1}$ surface-induced vibrational redistribution (IVR). For scattering from $\mathrm{Gr} / \mathrm{Ni}(111), v_{3} \rightarrow v_{1}$ surface-induced IVR is absent with all the scattered $\mathrm{CH}_{4}$ remaining in the $v_{3}$ state indicated by the fact that all lines that originate from the $v_{1}$ state are missing from the lower spectrum. (The spectral lines have a derivative form due to the use of frequency modulation for lock-in detection of the bolometer signal.)

bare $\mathrm{Ni}(111)$ surface, state-resolved BILT detection was used to quantify the rotational-state population with $J$ up to 8 as shown in Fig. 2.

Figures 2 and 3 show how for the same incident kinetic energy, scattering from $\mathrm{Gr} / \mathrm{Ni}(111)$ leads to stronger rotational excitation than scattering from bare $\mathrm{Ni}(111)$. Such a difference in rotational excitation could be caused by the larger corrugation of $\mathrm{Gr} / \mathrm{Ni}(111)$ compared to $\mathrm{Ni}(111)$ but other explanations such as differences in physisorption well depths cannot be excluded without theoretical modeling. Figure 3 shows rotational Boltzmann plots for the rotational distributions of Fig. 2. Except for the lowest $J$ levels, which correspond to (near-)elastic scattering, the rotational-state populations can be fitted by a Boltzmann distribution with a rotational temperature of $153 \pm 8 \mathrm{~K}$ for $\mathrm{Ni}(111)$ and $208 \pm$ $14 \mathrm{~K}$ for $\mathrm{Gr} / \mathrm{Ni}(111)$, much lower than the $673 \mathrm{~K}$ temperature

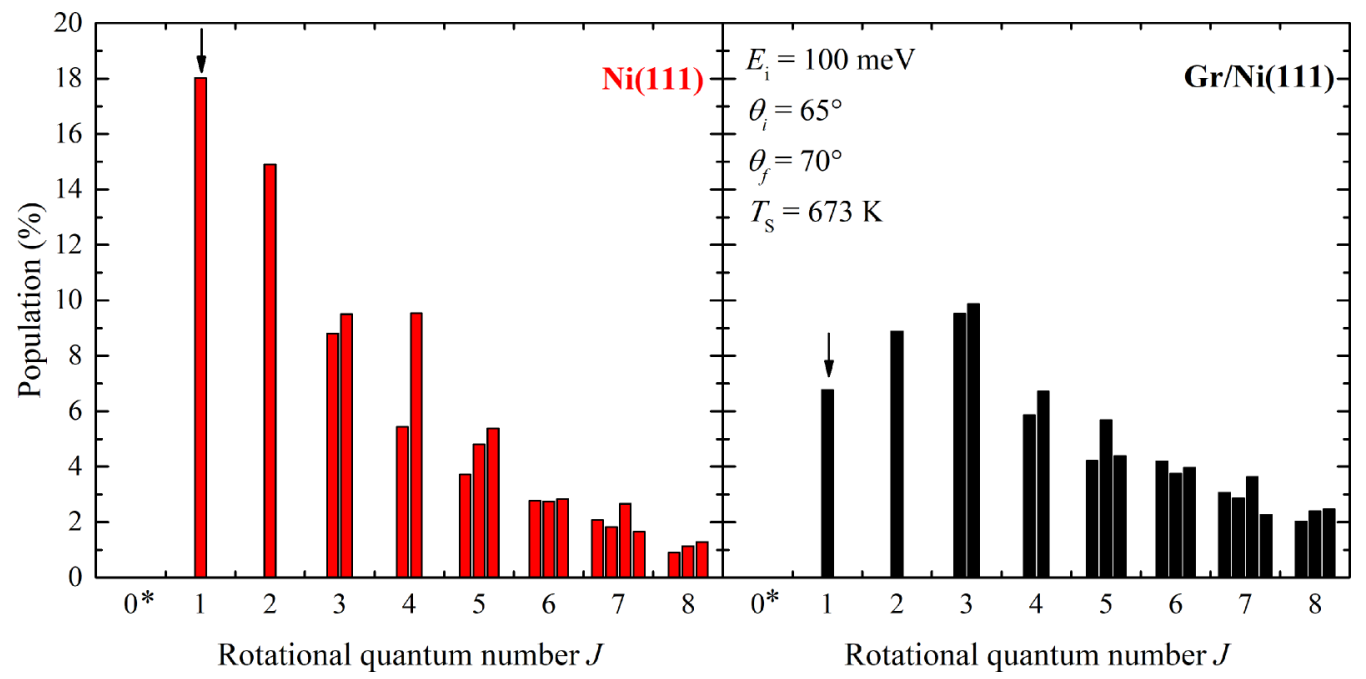

FIG. 2. Rotational level populations for $\mathrm{CH}_{4}\left(v_{3}, J\right)$ scattered from $\mathrm{Ni}(111)$ and $\mathrm{Gr} / \mathrm{Ni}(111)$. The incident $\mathrm{CH}_{4}$ was prepared in the $\left(v_{3}=1\right.$, $J=1$ ), state which is labeled by an arrow $\downarrow$. For $J>2$, multiple bars give the population of the individual sublevels for each value of $J$. Stronger rotational excitation is observed for scattering from $\mathrm{Gr} / \mathrm{Ni}(111)$ compared to bare $\mathrm{Ni}(111)$. (* For symmetry reasons, the $J=0$ level does not exist for the nuclear spin conformer of methane prepared in the incident beam.) 


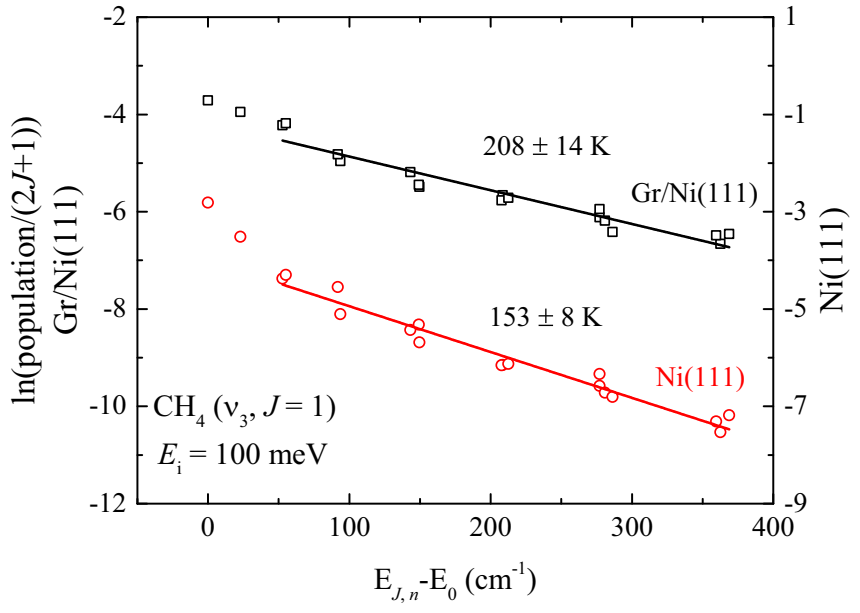

FIG. 3. Boltzmann fits for the rotational level populations shown in Fig. 2 as a function of rotational energy $E_{J, n}$ of the scattered $\mathrm{CH}_{4}$ for incident kinetic energy $E_{i}=100 \mathrm{meV}$ and a surface temperature of $673 \mathrm{~K} . E_{0}$ is the energy of the lowest rotational state $J=1$. Note the use of different vertical scales for $\mathrm{Gr} / \mathrm{Ni}(111)$ (left) and $\mathrm{Ni}(111)$ (right) for clarity of presentation. Solid lines are linear fits to the data excluding the two lowest rotational states $J=1$ and 2. The characteristic rotational temperatures given by the slopes of the linear fits are $153 \pm 8 \mathrm{~K}$ for $\mathrm{Ni}(111)$ and $208 \pm 14 \mathrm{~K}$ for $\mathrm{Gr} / \mathrm{Ni}(111)$.

of the target surface. The fact that both rotational temperatures are far below the surface temperature and that the scattering leads to nearly specular angular distributions [11] confirms that we are probing a fast and direct scattering process rather than a trapping-desorption channel in which case the incident molecules would thermalize with the surface before desorbing back into the gas phase.

Our calculations consider collisions at 16 points on the surface unit cell, reducible to 4 unique impact sites: the top, bridge, half-bridge, and near hollow sites [11]. Results for each site are shown in Fig. 4 for the case of a rigid lattice. We find that the $v_{3} \rightarrow v_{1}$ transition probability is the largest by far, that there is a strong variation in vibrational inelasticity with impact site, and that this correlates with the catalytic activity of the impact site. Density-functional theory (DFT) studies of methane dissociation on $\mathrm{Ni}$ and $\mathrm{Pt}$ surfaces find that the highest catalytic activity is over the top site [25], and that the barrier increases as we move towards the bridge site [15]. While we are well below these barriers at the incident energies considered here, our DFT calculations show that as the $\mathrm{CH}_{4}$ approaches a reactive site, the $\mathrm{C}-\mathrm{H}$ bond closest to the surface starts to elongate. The lower the barrier at a particular site, the sooner this increase in $\mathrm{C}-\mathrm{H}$ bond length starts to happen with respect to the distance above the surface.

In Fig. 4 we list the length of this $\mathrm{C}-\mathrm{H}$ bond for each impact site, at the point where the carbon is at a distance $Z_{C}$ above the surface such that $V_{0}\left(Z_{\mathrm{C}}\right)=0.2 \mathrm{eV}$; i.e., at the point of closest approach for a collision energy of $0.2 \mathrm{eV}$. We clearly see that the amount of $\mathrm{C}-\mathrm{H}$ stretching correlates with both catalytic activity and the magnitude of the $v_{3} \rightarrow v_{1}$ transition. This makes sense, since we are distorting the molecule close to the turning point, and this distortion, roughly a $\mathrm{C}-\mathrm{H}$ stretch, mixes the stretch normal-mode vibrations $v_{3}$ and $v_{1}$, which

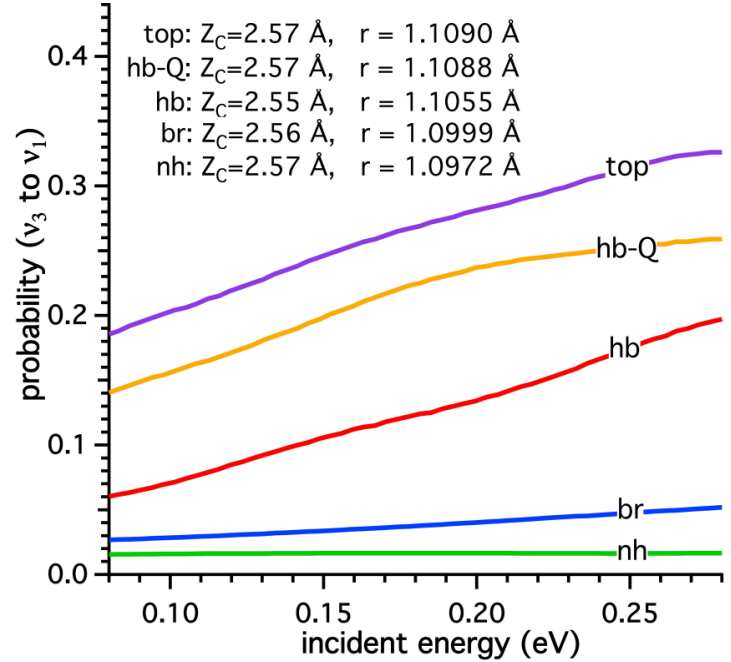

FIG. 4. Computed probabilities for the $v_{3} \rightarrow v_{1}$ transition as a function of energy, for impact at the top, bridge (br), half-bridge (hb), and near-hollow (nh) sites, and at the half-bridge site where the $\mathrm{Ni}$ atom is puckered by $0.1 \AA$ (hb-Q). Also shown are the distance of the carbon above the surface when $V_{0}=0.2 \mathrm{eV}, Z_{C}$, and the length of the reactive $\mathrm{C}-\mathrm{H}$ bond at this distance, $r$.

(classically) differ only by the relative phases of the $\mathrm{C}-\mathrm{H}$ stretching motion of the four bonds. At these low collision energies, we do not observe any bending distortions of the molecule near the turning point. Thus, we see only very small transition probabilities to the $2 v_{2}, 2 v_{4}$ and $v_{2}+v_{4}$ bending states. A recent calculation of $\mathrm{H}_{2} \mathrm{O}$ scattering from $\mathrm{Cu}(111)$ found a similar mixing of the two stretching vibrations [26]. While our study relates IVR in polyatomic-surface scattering with catalytic activity, a paper published over 30 years ago [27] predicted that the lengthening of the bond during a low-energy collision of $\mathrm{H}_{2}$ with a catalytically active surface could induce rovibrational transitions. Since then, a few other studies have noted how the existence of a dissociation channel can modify nonreactive diatom-surface scattering [28-30]. Finally, note that the site-to-site variation in the classical turning point, $Z_{C}$, is very small at $0.2 \mathrm{eV}$, and that the largest value of $Z_{C}$ is for the catalytically active top site. Thus the observed effects are not related to how close the molecule gets to the metal.

Lattice displacement changes the barriers to methane dissociation on metals, leading to strong temperature effects in dissociative chemisorption [31,32]. In Fig. 4 we plot results for impact at the half-bridge site, where the Ni atom closest to the methane is puckered out of the plane of the surface by $0.1 \AA$, roughly the rms vibrational amplitude of a $\mathrm{Ni}$ atom at $300 \mathrm{~K}$. On Ni(111), this puckering lowers the barrier by about $0.1 \mathrm{eV}$ [25], and we see that this lattice distortion significantly increases the $v_{3} \rightarrow v_{1}$ transition probability and the $\mathrm{C}-\mathrm{H}$ bond length, just as it increases the catalytic activity.

To more rigorously include these lattice motion effects, we would need to repeat these calculations, at all sites, for several values of the surface atom displacements, and then Boltzmann- and site average the results. However, we can estimate the total $v_{3} \rightarrow v_{1}$ transition probability for a rigid surface by averaging over the impact sites with their proper 
weighting [11], and we find that it is about $10 \%$ at 0.2 $\mathrm{eV}$. As in the experiments, the variation with energy is relatively weak for this near-resonant nonactivated transition, particularly given acceleration by the $0.2 \mathrm{eV}$ attractive well. By including lattice motion at the high temperatures of the experiment, our results in Fig. 4 suggest that this could easily increase by a factor of 2 or 3 , giving a branching ratio very similar to that in the experiments. A more quantative comparison with experiment would require a better treatment of rotation, and perhaps also $X$ and $Y$, since translational steering may be important at these low collision energies. Such a full 15-dimensional quantum treatment is not currently feasible.

To test our assertions, we repeat our calculations for the case where the $\mathrm{Ni}(111)$ surface is covered with a graphene overlayer. The methods for modeling this in a supercell DFT calculation are described elsewhere [33]. We considered methane impact at two sites: directly over a $\mathrm{C}$ atom that is directly over a $\mathrm{Ni}$ atom, and over the graphene hollow, which is over the $\mathrm{Ni}(111)$ hexagonal close-packed hollow. The graphene layer is kept rigid. The methane- $\mathrm{Gr} / \mathrm{Ni}(111)$ well depth is $0.26 \mathrm{eV}$ at both sites. We find that the $\mathrm{C}-\mathrm{H}$ bonds of methane are not elongated at all, suggesting that the surface is catalytically inactive, as expected. Moreover, at a collision energy of $0.15 \mathrm{eV}$, the $v_{3} \rightarrow v_{1}$ transition probability at both of these sites is only about $1.5 \%$, compared to $25 \%$ for the (bare) $\mathrm{Ni}(111)$ top site. These findings are consistent with our scattering experiments.
In conclusion, our state-to-state $\mathrm{CH}_{4}$ scattering experiments from bare and graphene-covered $\mathrm{Ni}(111)$ show that surface-induced IVR from the antisymmetric to the symmetric $\mathrm{C}-\mathrm{H}$ stretch normal mode occurs on the bare $\mathrm{Ni}(111)$ but is completely absent if a single layer of graphene is added to passivate the $\mathrm{Ni}(111)$ surface. Quantum scattering calculations reveal that the propensity for the observed $v_{3} \rightarrow v_{1}$ vibrational energy redistribution process is related to the extent of $\mathrm{C}-\mathrm{H}$ bond stretching during the molecule's closest approach to a scattering site, which in turn correlates with the catalytic activity of the specific surface site. Combined, the results of our experimental and theoretical work indicate that the probing of surface-induced IVR processes by state-to-state scattering experiments of polyatomic molecules from metal surfaces yields valuable information on the reactive potential-energy surface and the catalytic activity of the molecule-surface system under study. Extensions of this work to a wider range of experimental conditions are currently underway.

\section{ACKNOWLEDGMENTS}

The Lausanne group acknowledges financial support provided by the Swiss National Science Foundation (Grant No. 178775) and the Max Planck-EPFL Center for Molecular Nanoscience and Technology. We thank Vincent Boudon for spectroscopic data on hot-band transition for methane.
[1] L. B. F. Juurlink, D. R. Killelea, and A. L. Utz, State-resolved probes of methane dissociation dynamics, Prog. Surf. Sci. 84, 69 (2009).

[2] R. D. Beck and A. L. Utz, in Dynamics of Gas-Surface Interactions: Atomic-level Understanding of Scattering Processes at Surfaces, edited by R. D. Muiño and H. F. Busnengo (Springer, Berlin, 2012).

[3] R. D. Beck, P. Maroni, D. C. Papageorgopoulos, T. T. Dang, M. P. Schmid, and T. R. Rizzo, Vibrational mode-specific reaction of methane on a nickel surface, Science 302, 98 (2003).

[4] D. R. Killelea, V. L. Campbell, N. S. Shuman, and A. L. Utz, Bond-selective control of a heterogeneously catalyzed reaction, Science 319, 790 (2008).

[5] L. Chen, H. Ueta, R. Bisson, and R. D. Beck, Vibrationally bond-selected chemisorption of methane isotopologues on $\operatorname{Pt}(111)$ studied by reflection absorption infrared spectroscopy, Faraday Discuss. 157, 285 (2012).

[6] B. L. Yoder, R. Bisson, and R. D. Beck, Steric effects in the chemisorption of vibrationally excited methane on $\mathrm{Ni}(100)$, Science. 329, 553 (2010).

[7] T. E. Gough, R. E. Miller, and G. Scoles, Infrared laser spectroscopy of molecular beams, Appl. Phys. Lett. 30, 338 (1977).

[8] J. Werdecker, M. E. van Reijzen, B.-J. Chen, and R. D. Beck, Vibrational Energy Redistribution in a Gas-Surface Encounter: State-to-State Scattering of $\mathrm{CH}_{4}$ from $\mathrm{Ni}(111)$, Phys. Rev. Lett. 120, 053402 (2018).
[9] M. P. Schmid, P. Maroni, R. D. Beck, and T. R. Rizzo, Molecular-beam/surface-science apparatus for state-resolved chemisorption studies using pulsed-laser preparation, Rev. Sci. Instrum. 74, 4110 (2003).

[10] H. Chadwick, P. M. Hundt, M. E. van Reijzen, B. L. Yoder, and R. D. Beck, Quantum state specific reactant preparation in a molecular beam by rapid adiabatic passage, J. Chem. Phys. 140, 034321 (2014).

[11] See Supplemental Material at http://link.aps.org/supplemental/ 10.1103/PhysRevResearch.2.043251 for additional experimental and computational details.

[12] S. Nave, A. K. Tiwari, and B. Jackson, Dissociative chemisorption of methane on Ni and Pt surfaces: Mode-Specific chemistry and the effects of lattice motion, J. Phys. Chem. A 118, 9615 (2014).

[13] H. Guo and B. Jackson, Mode-selective chemistry on metal surfaces: The dissociative chemisorption of $\mathrm{CH}_{4}$ on $\mathrm{Pt}(111)$, J. Chem. Phys. 144, 184709 (2016).

[14] H. Chadwick, H. Guo, A. Gutierrez-Gonzalez, J. P. Menzel, B. Jackson, and R. D. Beck, Methane dissociation on the steps and terraces of $\mathrm{Pt}(211)$ resolved by quantum state and impact site, J. Chem. Phys. 148, 014701 (2018).

[15] B. Jackson, F. Nattino, and G. J. Kroes, Dissociative chemisorption of methane on metal surfaces: Tests of dynamical assumptions using quantum models and ab initio molecular dynamics, J. Chem. Phys. 141, 054102 (2014). 
[16] Z. J. Zhang, T. H. Liu, B. N. Fu, X. M. Yang, and D. H. Zhang, First-principles quantum dynamical theory for the dissociative chemisorption of $\mathrm{H}_{2} \mathrm{O}$ on rigid $\mathrm{Cu}(111)$, Nat. Commun. 7, 11953 (2016).

[17] B. Jiang and H. Guo, Quantum and classical dynamics of water dissociation on $\mathrm{Ni}(111)$ : A test of the site-averaging model in dissociative chemisorption of polyatomic molecules, J. Chem. Phys. 143, 164705 (2015).

[18] G. Kresse and J. Furthmuller, Efficient iterative schemes for ab initio total-energy calculations using a plane-wave basis set, Phys. Rev. B 54, 11169 (1996).

[19] G. Kresse and J. Hafner, Ab initio molecular-dynamics for liquid-metals, Phys. Rev. B 47, 558 (1993).

[20] G. Kresse and J. Hafner, Ab-initio molecular-dynamics simulation of the liquid-metal amorphous-semiconductor transition in germanium, Phys. Rev, B. 49, 14251 (1994).

[21] G. Kresse and J. Furthmuller, Efficiency of ab-initio total energy calculations for metals and semiconductors using a plane-wave basis set, Comput. Mater. Sci. 6, 15 (1996).

[22] G. Kresse and D. Joubert, From ultrasoft pseudopotentials to the projector augmented-wave method, Phys. Rev. B 59, 1758 (1999).

[23] D. Migliorini, H. Chadwick, F. Nattino, A. Gutierrez-Gonzalez, E. Dombrowski, E. A. High, H. Guo, A. L. Utz, B. Jackson, R. D. Beck, and G. J. Kroes, Surface reaction barriometry: Methane dissociation on flat and stepped transition-metal surfaces, J. Phys. Chem. Lett. 8, 4177 (2017).

[24] W. H. Miller, N. C. Handy, and J. E. Adams, Reaction-path Hamiltonian for polyatomic-molecules, J. Chem. Phys. 72, 99 (1980).
[25] S. Nave, A. K. Tiwari, and B. Jackson, Methane dissociation and adsorption on $\mathrm{Ni}(111), \mathrm{Pt}(111), \mathrm{Ni}(100), \mathrm{Pt}(100)$, and Pt(110)-(1 x 2): Energetic study, J. Chem. Phys. 132, 054705 (2010).

[26] L. Zhang and B. Jiang, Efficient Vibrational Energy Redistribution between Stretching Modes: State-to-State Quantum Scattering of $\mathrm{H}_{2} \mathrm{O}$ from $\mathrm{Cu}(111)$, Phys. Rev. Lett. 123, 106001 (2019).

[27] B. Jackson, Spectral grid study of ro-vibrational coupling in hydrogen-metal scattering, J. Phys. Chem. 93, 7699 (1989).

[28] C. T. Rettner, D. J. Auerbach, and H. A. Michelsen, Observation of Direct Vibrational Excitation in Collisions of $\mathrm{H}_{2}$ and $\mathrm{D}_{2}$ with a Cu(111) Surface, Phys. Rev. Lett. 68, 2547 (1992).

[29] M. del Cueto, X. Zhou, A. S. Muzas, C. Díaz, F. Martín, B. Jiang, and H. Guo, Quantum stereodynamics of $\mathrm{H} 2$ scattering from $\mathrm{Co}(0001)$ : Influence of reaction channels, J. Phys. Chem. C 123, 16223 (2019).

[30] R. Yin, Y. Zhang, and B. Jiang, Strong vibrational relaxation of NO scattered from $\mathrm{Au}(111)$ : Importance of the adiabatic potential energy surface, J. Phys. Chem. Lett. 10, 5969 (2019).

[31] A. K. Tiwari, S. Nave, and B. Jackson, The temperature dependence of methane dissociation on $\mathrm{Ni}(111)$ and $\mathrm{Pt}(111)$ : Mixed quantum-classical studies of the lattice response, J. Chem. Phys. 132, 134702 (2010).

[32] A. K. Tiwari, S. Nave, and B. Jackson, Methane Dissociation on Ni(111): A New Understanding of the Lattice Effect, Phys. Rev. Lett. 103, 253201 (2009).

[33] Q. Wang, L. Wei, M. Sullivan, S.-W. Yang, and Y. Chen, Graphene layers on $\mathrm{Cu}$ and $\mathrm{Ni}$ (111) surfaces in layer controlled graphene growth. RSC Adv. 3, 3046 (2013). 